\title{
Quaderni
}

QUADERNI Communication, technologies, pouvoir

\section{Entre revendication artiste et gramscisme de droite : le cinéma de Clint Eastwood comme apologie du libertarianisme américain}

\section{Gwendal Châton}

\section{(2) OpenEdition \\ Journals \\ Édition électronique \\ URL : http://journals.openedition.org/quaderni/863 \\ DOI : $10.4000 /$ quaderni.863 \\ ISSN : 2105-2956 \\ Éditeur \\ Les éditions de la Maison des sciences de l'Homme}

Édition imprimée

Date de publication : 5 janvier 2015

Pagination : $39-54$

Référence électronique

Gwendal Châton, «Entre revendication artiste et gramscisme de droite : le cinéma de Clint Eastwood comme apologie du libertarianisme américain », Quaderni [En ligne], 86 | Hiver 2014-2015, mis en ligne le 05 janvier 2017, consulté le 30 avril 2019. URL : http://journals.openedition.org/quaderni/863 ; DOI : 10.4000/quaderni.863 


\section{$D$ ossier}

\section{Entre} revendication artiste et gramscisme de droite :

le cinéma de Clint Eastwood comme apologie du libertarianisme américain

\section{Gwendal Châton}

Université d'Angers Centre Jean Bodin
«J'ai construit ma cabane. Lentement, à l'écart, en restant fidèle à mes principes. J'avance à mon rythme, personne ne me gouverne. ${ }^{1}$ "»

L'œuvre cinématographique de Clint Eastwood est aujourd'hui mondialement consacrée. Depuis le début des années 1990, la critique s'accorde à voir en lui le dernier des cinéastes classiques, l'héritier de John Ford, de Raoul Walsh et de Howard Hawks. Le public, quant à lui, considère qu'il est l'un des grands réalisateurs contemporains. Cette célébration désormais quasi unanime, particulièrement notable en France ${ }^{2}$, est pourtant advenue tardivement, après la sortie d'une quinzaine de films. L'une des raisons permettant de comprendre le « retard » de cette consécration est probablement - c'est notre hypothèse - de nature politique. En effet, Clint Eastwood a été longtemps décrit comme le héraut d'une révolution néoconservatrice : il est resté durablement prisonnier du personnage de L'inspecteur Harry (1971), un film de Don Siegel dénoncé à l'époque comme inspiré par un fascisme rampant. Cette interprétation a propagé l'image d'un acteur et d'un réalisateur adepte d'une sorte de gramscisme de droite ${ }^{3}$. C'est là, sans doute, ce qui explique qu'il s'est employé, depuis une vingtaine d'années, à prendre ses distances vis-à-vis de la politique pour affirmer une revendication avant tout artiste : il serait, selon ses dires repris par une partie de la critique, un pur esthète uniquement préoccupé par la narration et la mise en scène comme moyens incitant le spectateur à méditer le tragique de la condition humaine. Militant dévoué de la seule cause cinématographique, Clint Eastwood aurait ainsi déserté la politique. Une étude minutieuse de son œuvre incite cependant à douter de la réalité de ce récit ${ }^{4}$. Elle 
révèle aussi qu'il est vain de faire de lui, pour le blâmer ou pour le louer, une icône de la droite néoconservatrice. Car si cette œuvre foisonnante et éclectique possède bien une portée politique, c'est plutôt comme apologie d'une philosophie radicalement individualiste qu'il faut la lire : c'est bien le libertarianisme américain qui donne sa consistance à la « politique eastwoodienne ».

\section{Un libertarien à Hollywood}

Pour prendre la mesure de l'importance de la politique pour Clint Eastwood, il faut revenir sur son itinéraire. Sa trajectoire apparaît assez atypique à Hollywood, puisqu'il a toujours été un fidèle compagnon de route du parti républicain. Certes, quelques acteurs et réalisateurs affichaient avant lui leur sympathie pour le Grand Old Party - les moindres n'étant pas John Wayne ou Cecil B. DeMille. Mais ils restaient finalement peu nombreux dans un microcosme social majoritairement acquis à la cause démocrate ${ }^{5}$. Si les rangs des soutiens hollywoodiens du parti républicain sont aujourd'hui plus fournis, l'engagement de Clint Eastwood apparaît singulier en raison de sa précocité et de sa constance. C'est en 1951 que ce jeune acteur alors totalement inconnu choisit le camp républicain. Il reconduit ainsi l'identification partisane de ses parents, des « cols blancs » d'origine modeste appartenant à la frange inférieure de la middle class. À partir de 1952, Eastwood vote sans faillir pour tous les candidats républicains à la présidence. En 1968, alors que la « trilogie du dollar » de Sergio Leone ${ }^{6}$ l'a sorti de l'anonymat et enrichi, il apporte avec discrétion un soutien financier à la campagne présidentielle de Richard Nixon. Ce n'est qu'en 1971, alors qu'il vient d'achever son premier long-métrage, qu'il fait son coming out politique : il explique dans le Washington Post pourquoi, dans le contexte de l'enlisement au Vietnam, il soutient Nixon. Désormais, plus personne ne peut ignorer les orientations politiques de celui qui incarne au même moment le très controversé Dirty $\mathrm{Harry}^{7}$. Par la suite, il n'hésitera pas à mettre régulièrement son image et sa fortune au service des candidats de son parti.

Dans cet itinéraire, les années 1980 correspondent à un moment particulier. Ce sont les années durant lesquelles son « candidat du cœur », l'ancien acteur, ancien démocrate et ancien gouverneur de Californie Ronald Reagan, va exercer le pouvoir. Ces années s'ouvrent sur la célèbre proclamation reaganienne selon laquelle « in this present crisis, government is not the solution to our problem ; government is the problem ${ }^{8} »$. Les années Reagan coïncident avec l'acmé de l'engagement politique du réalisateur : il est à la fois personnellement proche du président et politiquement proche des reaganomics. Il cède même à son tour à la tentation politique : en 1986, il brigue la mairie de Carmel-by-the-Sea, une petite ville d'artistes située en Californie. L'expérience n'est cependant pas très concluante : une fois la mairie conquise, grâce à une campagne très professionnelle dans laquelle il a investi beaucoup d'argent, la gestion quotidienne de la ville ne l'intéresse rapidement plus. Il ne se représente d'ailleurs pas et les rumeurs qui lui prêtaient le projet d'entrer en politique au niveau national sont rapidement démenties. En revanche, les interprétations qui font de ses films des vecteurs plus ou moins subtils du reaganisme en sortent renforcées. Ainsi de l'un de ses biographes, Patrick McGilligan, qui affirme qu'à cette époque, « le flux politique de sa car- 
rière allait se refléter, plus que jamais, dans des films à l'idéologie proche du républicanisme ${ }^{9}{ }$.

Après le départ de Reagan, le réalisateur semble prendre ses distances avec la politique partisane. S'il soutient encore George Bush en 1988, il dit avoir voté pour le libertarien soft, Ross Perot, en 1992 : c'est sa première infidélité au parti républicain en quarante ans. En 1996, alors qu'il tourne Les pleins pouvoirs (1997), il évite soigneusement de prendre parti en faveur du GOP. McGilligan peut ainsi soutenir que durant cette décennie "Clint abandonna peu à peu l'activisme politique ${ }^{10} »$. Il faut dire que 1992 constitue un tournant dans sa seconde carrière de réalisateur : il reçoit en effet deux Oscars - meilleur réalisateur et meilleur film pour son « western révisionniste » Impitoyable $(1992)^{11}$. L'heure de la reconnaissance de son statut d'auteur a enfin sonné. Et tout indique que la consécration tant attendue le conduit à s'éloigner de la politique pour cultiver l'image d'un réalisateur désengagé situé au-dessus de la mêlée. Pour autant, cet éloignement sera de courte durée : durant les années 2000, il soutient George W. Bush puis John McCain. Mais c'est surtout son intervention à la Convention de Tampa, en août 2012, en faveur de Mitt Romney qui incite à remettre en cause la sincérité de cette prise de distance à l'égard de la politique ${ }^{12}$.

Ce vieux compagnon de route du parti républicain n'a cependant jamais été un conservateur oldfashioned ou new style $e^{13}$. Au sein du GOP, Clint Eastwood doit en effet être rattaché au courant libertarien. C'est ce qu'a bien perçu l'un de ses critiques américains récents qui le rapproche de la grande figure du libertarianisme : la roman- cière et essayiste d'origine russe Ayn Rand ${ }^{14}$. Le libertarianisme est une philosophie politique mal connue en France ${ }^{15}$. Elle a pour particularité de refuser de se situer sur le très classique axe gauche/droite. Les libertariens se positionnent ainsi à la fois à gauche pour les libertés personnelles et à droite pour les libertés économiques. Ils se livrent surtout à une défense tous azimuts de la liberté individuelle contre l'ensemble des formes de domination, qu'elles soient le fait de l'État ou de la société. Le libertarianisme associe ainsi le libéralisme économique le plus farouche au libéralisme culturel et moral le plus résolu. En un mot, il fait primer la liberté-indépendance sur la liberté-capacité d'action ${ }^{16}$. Cette défense de la liberté s'étend aussi au domaine des relations internationales : les libertariens sont plutôt pacifistes et partisans d'un isolationnisme forcené, ce qui les distingue là encore très clairement des néoconservateurs. Cette philosophie politique correspond ainsi à une radicalisation de l'individualisme souvent associé aux États-Unis.

Cette sensibilité, qui voit s'affronter plusieurs courants, est le socle d'un mouvement qui a fini par s'autonomiser dans les années 1970, après avoir cheminé un temps avec les conservateurs, puis avec les liberals $^{17}$. Les libertariens se sont regroupés en 1971 au sein du Libertarian Party et autour de nombreux think tanks, le plus influent étant le Cato Institute. Pour autant, beaucoup de libertariens sont restés dans le giron du parti républicain : c'est là que l'on trouve les partisans les plus « instinctifs » et les moins « théoriciens » qui sont si nombreux aux États-Unis. C'est le cas de Clint Eastwood, dont l'adhésion au libertarianisme s'est nettement affirmée au fil du temps sans que cela ne le 
conduise à rompre avec le GOP. Dans les années 1970 et 1980 en effet, son libertarianisme n'est encore que balbutiant et le réalisateur s'en tient encore souvent à des positions conservatrices : il critique à échéance régulière le développement d'une "société d'assistés » et explique que le gouvernement doit avant tout inciter les individus à faire quelque chose de leur vie ${ }^{18}$. Il va ensuite se structurer quand Eastwood prend momentanément ses distances avec le GOP, pour devenir explicite dans les années 2000.

Cette évolution est attestée par quelques déclarations récentes. En 2008, il se définit comme un « libertarien de toujours » : «I mean, I've always been a libertarian. Leave everybody alone. Let everybody else do what they want. Just stay out of everybody else's hair. ${ }^{19}$ " En 2011, il affirme que sa philosophie politique repose sur un principe simple : «Let's spend a little more time leaving everybody alone. Just give everybody the chance to have the life they want. ${ }^{20}$ » S'ajoute à ces propos le fait que Clint Eastwood a toujours défendu, contre le Big government, des positions clairement libertariennes : il s'est ainsi prononcé en faveur d'une liberté d'expression totale, de la libéralisation des drogues, de la liberté sexuelle, de l'avortement, de l'euthanasie ou encore du mariage homosexuel. Il s'est aussi déclaré contre la peine de mort et contre toutes les formes de racisme $^{21}$. Il est enfin un athée revendiqué qui, dans un pays aussi religieux que les États-Unis, n'hésite pas à décrire les religions comme des carcans oppressants ${ }^{22}$. De l'analyse de cet itinéraire, il faut conclure que Clint Eastwood a toujours cherché à vivre conformément au dogme libertarien : «Minimum Government,
Maximum Freedom ${ }^{23}$ ». D'où une question difficile à contourner : ces convictions imprègnent-elles, d'une manière ou d'une autre, son œuvre ? Pour y répondre, on peut partir de sa réflexion sur l'histoire de l'Amérique.

\section{Un plaidoyer pour une Amérique déclinante}

Le cinéma de Clint Eastwood doit avant tout être regardé comme une interrogation sur le destin de l'Amérique qui conduit à une défense passionnée du credo américain ${ }^{24}$. Conscient de l'ambition démesurée qui est à la base de son histoire l'Amérique est pour lui «l'idée la plus folle de l'Histoire ${ }^{25} »-$, il a construit son œuvre comme un plaidoyer pour une nation qui, depuis la fin des années 1960, questionne anxieusement son identité ${ }^{6}$. Cette réflexion sur l'histoire nationale remonte jusqu'à la conquête de l'Ouest. À cet égard, nul doute que Clint Eastwood a été profondément marqué par le genre de l'americana, ces grandes sagas sur l'expérience des pionniers : en ce sens, il est bien un héritier de John Ford. Mais au-delà de cette influence, il renouvelle ce genre en livrant une réflexion personnelle sur l'histoire nationale dont le fil rouge est la question de la violence.

Une idée-force structure l'ensemble de son œuvre : si l'Amérique s'est construite sur un rejet des vices de la vieille Europe, elle n'a pas réussi à éradiquer une violence qui a accompagné toute son histoire. Trois exemples qui tiennent à l'époque de la fondation des États-Unis permettent de mettre en évidence la centralité de ce thème. Dans Pale Rider (1985), une très pieuse et très pacifique communauté de chercheurs d'or se donne pour but de fonder une ville sur la 
base des valeurs évangéliques. Mais elle en est empêchée par la terreur que fait régner le riche propriétaire de la cité voisine : dès l'origine, la passion du lucre et l'hubris viennent barrer la route des pionniers. Dans Josey Wales hors-la-loi (1976), qui sort au moment du bicentenaire de l'Indépendance, la guerre de Sécession est décrite comme un moment tragique de «brutalisation » de la société américaine ${ }^{27}$. Dans L'homme des hautes plaines (1973) enfin, son premier « western révisionniste ", l'action se situe dans le Far West des pionniers, mais l'ambiance étrange de ce film suggère qu'elle pourrait se tenir partout et en tout temps. Souvent considéré à tort comme une apologie de la violence, il est en fait construit sur une thèse anthropologique forte : toute civilisation se construit sur une violence fondatrice qui correspond au meurtre d'une victime émissaire ${ }^{28}$. Et pour Clint Eastwood, il est clair que l'Amérique ne fait pas exception à la règle.

Cette réflexion pessimiste sur la violence, qui en affirme la fatalité, a immanquablement donné prise à des interprétations fautives : on a souvent accusé Eastwood de l'esthétiser dans le but de la légitimer. Cette thèse est cependant difficile à soutenir après Impitoyable. Dans ce film, le réalisateur concentre son propos sur trois messages : la violence est tapie en chacun de nous; elle est fondamentalement irréductible ; toute violence est au fond impardonnable - le titre original du film est Unforgiven. Le personnage central, une sorte d'anti-héros, est un gunfighter repenti qui s'accroche au souvenir de sa femme, à sa ferme et à ses deux enfants pour tenir à distance son passé. Mais à la faveur des circonstances, il redevient la brute sanguinaire qu'il n'a jamais cessé d'être et se livre à un massacre qui constitue un terrible retour du refoulé2 ${ }^{2}$. En face de lui, le shérif censé incarner la loi est lui aussi un ancien tueur qui masque mal sa véritable nature. Métaphoriquement, il se montre incapable de construire sa propre maison qui, selon l'heureuse métaphore d'un personnage, n'a pas un seul angle droit. Sa bestialité foncière se révèle dans une scène-clé : il y passe à tabac un tueur à gages tout juste arrivé en ville en lui expliquant que quand il le frappe, il ne fait que lui parler. Et cette scène essentielle se déroule symboliquement un 4 juillet : on comprend ici que la nation américaine est fondée sur une violence qui accompagne toute son histoire comme une malédiction.

L'Amérique contemporaine n'est pas épargnée par cette fatalité. Plusieurs films dont l'action est située au $\mathrm{XX}^{\mathrm{e}}$ siècle ont pour arrière-plan un événement particulièrement traumatique : l'assassinat du président Kennedy ${ }^{30}$. C'est dans Un monde parfait (1993) que la thématique de la violence fait l'objet du traitement le plus approfondi. Butch, un truand en cavale, a été poussé du mauvais côté par les hasards de la naissance et les circonstances de la vie, mais il est aussi capable d'être une figure paternelle bienveillante et protectrice, une sorte de père de substitution pour l'enfant qu'il a pris en otage après son évasion. Son poursuivant est un Texas Ranger solidement arrimé à ses grands principes, mais on comprend qu'il est aussi celui qui a participé à l'évolution néfaste de Butch en le faisant jadis placer en maison de correction, c'est-à-dire en le faisant entrer à l'école du crime. L'agent du FBI est quant à lui parfaitement corrompu : il passe son temps à harceler sexuellement les femmes et il finit par tuer Butch hors de toute légalité et de toute nécessité. Enfin, l'enfant innocent devient 
un tueur, comme Butch avant lui, en cherchant à défendre les faibles et les opprimés. Un monde parfait est ainsi l'aboutissement magistral du projet déconstructionniste d'Eastwood dont le but est de rompre avec tout manichéisme simpliste. Ce film se clôt d'ailleurs sur un constat désabusé de Red Garnett, le Ranger pourtant très rustre, qui lâche un laconique : « Je ne sais pas. Je ne sais rien du tout. » Novembre 1963 est ce moment charnière de l'histoire américaine dont le sens est révélé par ce propos laconique, un tournant caractérisé par un écroulement du sol des évidences. Après cette date, le pays est entré dans une période d'incertitude qui s'apparente à une longue crise morale.

Cette violence omniprésente fait l'objet d'une condamnation assumée en fait bien avant Impitoyable, depuis au moins Bronco Billy (1980). La scène dans laquelle le héros de ce film, une piteuse copie de Buffalo Bill, est provoqué en duel par un shérif goguenard est éloquente : en jetant son arme à terre, il préfère être humilié plutôt que relever le gant. Dernièrement, c'est dans Gran Torino (2008) qu'Eastwood a donné à cette idée toute son ampleur. Le personnage de Walt Kowalski n'est pas un doux : il n'hésite pas à sortir sa carabine pour défendre son jardin. Mais le film illustre clairement le danger qu'il y a à entrer dans la spirale de la violence : c'est à partir du moment où il s'en prend à l'un des membres du gang qui persécute son jeune ami Hmong que s'enclenche la mécanique implacable qui va conduire au viol de la jeune Sue. Conscient de son erreur, Kowalski enferme Thao pour l'empêcher de prendre part à l'affrontement final et il s'offre en victime expiatoire. Avant d'aller trouver la mort, il lui donne la médaille de bra- voure qu'il a gagnée pendant la guerre de Corée et prend soin de lui expliquer que le véritable courage consiste parfois à refuser le combat. Un message complexe structure ainsi le discours du « dernier Eastwood ». D’un côté, le monde est décrit comme étant le lieu d'un struggle for life, comme l'illustre Million Dollar Baby (2004) : Frankie répète à Maggie de manière obsessionnelle de toujours tenir sa garde, car une seconde d'inattention suffit pour tout perdre. Mais de l'autre, Eastwood explique que la loi du talion n'est jamais une solution : dans Invictus (2009), c'est par la tolérance et le pardon que l'avenir de la nation peut être construit. Au fil des années, le cinéma de Clint Eastwood semble ainsi s'affirmer comme une œuvre habitée par un humanisme fondamental.

Cette réflexion sur la violence ne pouvait éviter d'aborder la guerre. C'est le diptyque tardif Mémoires de nos pères/Lettres d'Iwo Jima (2006) qui prend ce thème à bras le corps. Son message est limpide : on ne fait pas la guerre pour sa patrie, mais pour sauver sa peau et celle de ses amis. Le patriotisme n'est qu'une illusion entretenue par l'État pour servir ses propres intérêts. De plus, la guerre voit s'affronter des camps qui, au-delà des différences culturelles, comportent autant d'humanité que d'inhumanité. Des deux côtés, on rencontre des individus qui tentent uniquement de se soustraire aux contraintes que font peser sur eux l'État et la société. Ainsi du jeune boulanger Saigo qui, dans Lettres d'Iwo Jima, incarne cet individu qui ne pense qu'à rentrer vivant pour retrouver sa femme et son fils né après son départ, et à aider les amis qu'il s'est faits au front. C'est pourquoi il refuse obstinément de se conformer à l'éthique japonaise traditionnelle qui 
bannit la reddition et prône le suicide. Ce film profondément pacifiste montre que la réflexion eastwoodienne sur la violence finit par déborder l'histoire américaine pour conduire à une interrogation plus ample sur la nature humaine. Cet élément reste cependant incontournable pour qui veut comprendre la portée politique du cinéma d'Eastwood : c'est en effet à partir de cette méditation lucide, à laquelle s'ajoute le constat de désappointement idéologique de l'Amérique, que le réalisateur va déployer une « politique eastwoodienne » qui hésite entre rébellion anarchiste et résignation démocratique.

\section{Entre rébellion anarchiste et résignation démocratique}

Face à cette permanence de la violence, comment le libertarien Eastwood envisage-t-il le rôle de l'État? Est-il une garantie efficace de la paix civile ou, au contraire, le vecteur d'une insupportable coercition? Doit-on en faire une cause de la crise contemporaine ou constitue-t-il, à l'inverse, une planche de salut ? Il est difficile de donner une réponse tranchée à ces questions. Une chose est certaine néanmoins, c'est que son cinéma développe une vision plutôt dépréciative du Léviathan qui plonge ses racines dans la tradition de l'anarchisme individualiste d'un Henry David Thoreau, d'un Lysander Spooner ou d'un Benjamin Tucker. En effet, l'État y est toujours étroitement associé à la violence et à la corruption ; le monde politique y est décrit comme un repère de politiciens égoïstes, cyniques et timorés.

Dans Josey Wales hors-la-loi, le personnage du sénateur est une incarnation parfaite du politicien sans scrupules : il promet la liberté aux partisans sudistes qui déposeront les armes, mais il les fait exécuter à la mitraillette dès qu'ils sont désarmés. Dans Mémoires de nos pères, les dirigeants sont présentés comme des lâches préservés de l'horrible réalité de la guerre et uniquement soucieux de propagande. Mais c'est probablement dans Les pleins pouvoirs que la critique de l'État et de la politique se fait la plus systématique. Eastwood y dépeint un président américain machiavélique : il est obsédé par le sexe et porté sur la boisson, c'est-à-dire dominé par ses pulsions ; il ment à longueur de temps et avec une déconcertante facilité, en privé comme en public, à ses amis et à ses collaborateurs comme au peuple; il est prêt à tout, même à ordonner un meurtre, pour se maintenir au pouvoir. La proximité avec Lord Acton, qui expliquait que «le pouvoir tend à corrompre, et le pouvoir absolu corrompt absolument», ou encore que «les grands hommes sont presque toujours des grands méchants », est frappante ${ }^{31}$. Clint Eastwood ajoute même à ces postulats pessimistes que « the hunger for absolute power and, more to the point, the abuse of power, are part of human nature ${ }^{32} »$. Au-delà de ce film, le réalisateur déploie avec constance une vision négative de la politique qui part de l'État pour s'étendre aux principales institutions sociales.

Beaucoup d'institutions semblent en effet contaminées par cette corruption de l'ordre politique, comme si cette dernière ne faisait que révéler une corruption de la société moderne dans son ensemble. Il faut dire que ces institutions apparaissent atteintes d'une même maladie : la bureaucratisation. Ce phénomène, pointé très tôt par Max Weber, aurait donné naissance à une société opprimant les individus. Dans les années 
1980, à l'unisson des doctrines reaganienne et thatchérienne, Clint Eastwood explique ainsi que «l'emprise de la bureaucratie s'accroît à mesure que la planète se rétrécit et que les problèmes de société se compliquent», à tel point qu'il redoute "que l'indépendance individuelle ne devienne un rêve démodé33 $»$. C'est ce qu'exprime son cinéma qui décrit des univers sociaux gangrenés par la bureaucratie. C'est le cas de la police dans Dirty Harry ou de l'armée dans Le maître de guerre (1986), deux institutions régaliennes étouffant l'initiative individuelle sous la tyrannie des procédures.

Ce diagnostic « sociologique » se double d'une condamnation morale. Dans La Sanction (1975), le réalisateur renvoie dos-à-dos les deux camps de la guerre froide au motif que leurs services d'espionnage sont truffés des mêmes agents foncièrement cupides et amoraux : symboliquement, le dénommé Dragon qui travaille pour les États-Unis a jadis collaboré avec les nazis. Cette égalité dans le vice est d'ailleurs ce qui a conduit le héros, Jonathan Hemlock, à retourner enseigner l'histoire de l'art à l'Université - une des rares institutions jamais attaquées par Clint Eastwood. Dans J. Edgar (2011), c'est la CIA qui fait l'objet d'une description peu amène : elle est présentée comme l'enfant monstrueux d'un mythomane paranoïaque. Mais c'est sans doute avec L'échange (2008) que cette critique anti-institutionnelle est le plus amplement développée : l'héroïne est une femme célibataire qui, avec pour seules ressources son intelligence et sa pugnacité, doit se battre contre un maire manipulateur, une police oppressive, une justice corrompue, une institution médicale liberticide, des médias sans scrupules et un conformisme social culpabilisant.
Le personnage de Christine Collins est le symbole de la lutte de l'individu contre une société qui s'emploie méthodiquement à le broyer : elle est un authentique héros libertarien, semblable à ceux que l'on rencontre dans les romans d'Ayn Rand ${ }^{34}$.

Cette vision de la politique et de la société est le produit d'une inclination anarchiste évidente, bien que rarement explicite. C'est sans doute dans une scène-clé de Josey Wales hors-la-loi, le chef-d'œuvre libertarien de Clint Eastwood, qu'elle est formulée le plus clairement. Il s'agit du long dialogue entre Josey Wales et le chef indien Grand Bison. Le héros cherche alors à obtenir son accord pour installer une ferme dans une zone retirée située en territoire comanche, et ainsi mettre fin à la dérive meurtrière qu'il poursuit depuis que sa famille a été massacrée par des partisans nordistes. Voici ce dialogue, sorte de manifeste de l'anarchisme individualiste dans sa version Far West :

Josey Wales : «C'est avec toi que je viens mourir. Ou que je viens vivre. Ce n'est rien de mourir pour toi ou pour moi. C'est vivre qui est dur, quand tous ceux qu'on a aimés ont été violés et massacrés. Les gouvernements ne vivent pas ensemble comme les hommes. Un gouvernement ne t'offre pas le pacte ou le combat régulier. Moi, je viens pour t'offrir l'un ou l'autre. Pour recevoir l'un ou l'autre de toi. Tel que tu me vois, tu sais que ma parole de mort est sincère. Et que ma parole de vie aussi est sincère. (...) Je viens pour l'une ou pour l'autre. (...) Je ne te promets aucune autre richesse. C'est seulement la vie que je t'offre et seulement la vie que je demande. Et je dis moi que les hommes peuvent vivre ensemble sans se massacrer ou se haïr. » 
Grand Bison : "Je sais que les gouvernements ont tous des chefs à la langue fourchue. Il y a du fer dans tes paroles de mort que les Comanches entendent. Alors, il y a du fer aussi dans tes paroles de vie. Aucun papier ne peut contenir le fer. Le fer doit être dans l'homme. La parole du Grand Bison contient aussi le fer de la mort et de la vie. Il est juste que les guerriers que nous sommes se mesurent dans la lutte pour la vie ou la mort. Nous sommes pour la vie. »

Cette lutte pour la reconnaissance s'achève sur la conclusion d'un pacte de sang entre deux hommes qui vivent leur liberté non pas par l'État, mais contre l'État ${ }^{35}$.

Si l'œuvre d'Eastwood révèle d'incontestables penchants anarchistes, la vision de la politique qu'elle véhicule n'est pas toujours aussi radicale et on peut également y déceler certains signes d'engagement démocratique. Pour le comprendre, il faut faire converger le questionnement sur la violence et l'interrogation sur la politique. Pour contenir une violence omniprésente, la démocratie se présente en effet comme une solution commode : elle institutionnalise la gestion pacifique des conflits, elle instaure la modification des titulaires du pouvoir sans recours à la violence et elle ouvre la possibilité d'une destitution des dirigeants. Comme l'explique le cinéaste : "Une des grandes vertus du système démocratique, c'est que s'ils s'égarent vraiment trop, on les débarque. ${ }^{36}$ " Dès Magnum Force (1973), l'inspecteur Harry Callahan proclamait d'ailleurs un soutien - il est vrai seulement par défaut - à la démocratie : "Je déteste ce vieux système, mais tant que l'on ne m'en proposera pas un qui soit plus convaincant, je m'y tien- drai. » S'il n'appartient pas à l'espèce des démocrates béats, Clint Eastwood envisage néanmoins la démocratie comme un moyen utile permettant d'assurer un objectif que Montesquieu a placé très tôt au cœur du libéralisme : la sûreté ${ }^{37}$. Il faudra attendre le « dernier Eastwood » pour qu'il reconnaisse à ce système une véritable positivité, mais à la condition que le peuple sache se doter d'un leader charismatique : dans Invictus, le traitement de la figure de Nelson Mandela montre que le réalisateur reste prisonnier du mythe de l'homme providentiel.

Cette résignation démocratique n'en demeure pas moins très souvent subvertie par un tropisme anarchiste. Il semble en effet que pour sortir des impasses de la modernité et pour relancer l'odyssée américaine, le véritable espoir réside dans le recours à des petites communautés reposant sur l'association volontaire : c'est au sein de telles communautés que les individus peuvent espérer trouver le bonheur dans la liberté. C'est donc par le recours à une vision mythifiée de l'Amérique des origines que Clint Eastwood peut proposer une utopie fournissant un nouvel horizon à la patrie des Founding Fathers ${ }^{38}$.

\section{La communauté libertarienne comme horizon d'utopie}

On doit à Ferdinand Tönnies l'opposition classique entre communauté et société ${ }^{39}$. Si Émile Durkheim voyait dans la première «le communisme porté à son plus haut point de perfection $^{40}$ », c'est pourtant vers elle que se tourne Clint Eastwood. Sa conception de la communauté est cependant très différente de celle des deux sociologues. Elle est pour lui avant tout un groupe 
humain reposant sur la libre association d'individus cherchant à maximiser leur liberté. La société étant toujours peu ou prou le lieu d'une oppression de l'individu, elle doit être concurrencée par la multiplication de petites communautés permettant à chacun de mener librement son « plan de $v^{4} e^{41} \gg$. C'est par la célébration de la communauté librement choisie que le libertarianisme de Clint Eastwood trouve à s'exprimer.

L'individualisme défendu par le réalisateur présente toutefois deux singularités notables. Tout d'abord, l'individu eastwoodien n'a pas de sexe. Contrairement aux préjugés qui font de lui un défenseur de la domination masculine, ses films montrent qu'hommes et femmes sont également frappés par l'oppression et qu'ils sont également condamnés à lutter pour leur liberté. Qu'il suffise de rappeler la place centrale occupée par les personnages féminins dès Josey Wales hors-la-loi, L'épreuve de force (1977) ou Le retour de l'inspecteur Harry (1983), et par la suite dans Sur la route de Madison (1995), Million Dollar Baby, L'échange ou Gran Torino. Ensuite, cet individualisme ne s'accompagne pas d'une valorisation de la réussite matérielle. Dans Pale Rider, ce message est explicite dans la scène durant laquelle les chercheurs d'or se réunissent afin d'examiner la proposition de rachat de leur terre faite par celui qui les persécute. Le dilemme se présente de la manière suivante : soit ils acceptent cette offre, gagnent à la fois argent et tranquillité, mais doivent renoncer à leur rêve ; soit ils la rejettent, restent pauvres et persécutés, mais conservent leur idéal. Alors que le mystérieux preacher refuse d'orienter leur choix, ils finissent par se rallier au discours de Hull Barret qui les convainc de refuser l'offre en utilisant un argument simple : l'or n'est pas une fin en soi, c'est seulement un moyen qui doit permettre à chacun de fonder une famille et à tous de créer une ville et bâtir une église. On ne s'étonnera donc pas qu'Eastwood, lors de la promotion du film, ait déclaré ceci : «Il y a dix ans, les jeunes se promenaient pieds nus avec des colliers de fleurs au cou. Aujourd'hui, le pendule est reparti très loin dans l'autre sens, avec cette génération qu'il est convenu d'appeler les yuppies : ceux pour qui tout se résume dans le fait de gagner beaucoup d'argent tout de suite, d'avoir la meilleure chaîne stéréo, ceux qui se sentiraient déshonorés s'ils ne roulaient pas en BMW, les jeunes piranhas du matérialisme qu'on voit tenir le haut du pavé depuis quelques années. Leur obsession du "chacun pour soi" n'a rien à voir avec les valeurs individuelles que je défends. Entre les hippies et les yuppies, on doit quand même pouvoir trouver un moyen terme ! ${ }^{42}{ }^{\prime}$

De toute évidence, ce sont les communautés libertariennes qui permettent de trouver ce moyen terme. Les héros de ses films donnent en effet souvent naissance à des versions baroques du type de communauté proposé par Ayn Rand au milieu des années $1950^{43}$. Ces communautés constituent par définition un refus en acte de l'égoïsme, mais elles visent simultanément à ce que personne ne sacrifie au groupe sa liberté et sa singularité. Ce motif irrigue l'ensemble de l'œuvre d'un réalisateur qui affirmait très tôt que « la bureaucratisation de la société, l'expansion tentaculaire de l'État, ont incité les individus à former des contre-sociétés ${ }^{44} \gg$. On se contentera d'en donner deux exemples. Dans Josey Wales hors-la-loi, la fuite du héros s'accompagne de la formation d'une troupe qui agrège un vieux chef cherokee « civilisé », mais sans illusions 
sur la " civilisation » des blancs, une jeune indienne navajo violée et réduite en esclavage, une jeune fille simple d'esprit et sa vieille mère acariâtre - des nordistes alors que le héros était un partisan sudiste-, deux immigrés mexicains, une prostituée et un vieux chien galeux. À l'issue de l'épisode déjà cité, Josey Wales réussit à installer ce petit groupe dans un ranch abandonné, à l'écart des villes corruptrices et de l'autorité de Washington. Tous ses membres souhaitent y reconstruire leurs vies brisées en rejouant l'aube de l'Humanité. Au moment du bicentenaire de l'Indépendance, après le choc du Watergate et le désastre du Vietnam, Clint Eastwood rappelle ainsi aux Américains d'où ils viennent afin de favoriser un nouveau départ.

On trouve une autre contre-société libertarienne intéressante dans Million Dollar Baby : la salle de boxe miteuse de Frankie est décrite comme le lieu d'une vie authentique où l'on croise un vieil entraîneur misanthrope et bougon hanté par la culpabilité, un ancien boxeur borgne mais clairvoyant, un jeune illuminé rachitique qui délire sur une carrière de champion, des arrivistes qui trahiront leur entraîneur pour parvenir et enfin une jeune fille pauvre et pure qui cherche à échapper à sa condition. Ayant choisi la boxe, elle convainc non sans mal ce vieil entraîneur misogyne de lui enseigner son art, devient une championne à force de travail, puis sombre dans la déchéance physique après un mauvais coup encaissé pour avoir baissé sa garde durant quelques secondes. Entre-temps, elle a cependant réussi à s'extraire d'une famille sans scrupules pour trouver une place dans la communauté de Frankie. Elle a aussi réalisé son rêve en affirmant sa liberté, qui culminera dans son choix du suicide assisté. Ces deux communautés peuvent être dites libertariennes pour plusieurs raisons : elles se construisent à la fois contre l'État et contre la société ; elles naissent d'un choix libre et révocable; elles sont le lieu d'une solidarité uniquement choisie; elles assurent à la fois l'autonomie du groupe et la liberté des individus qui le composent. L'appartenance à ces communautés repose sur un choix individuel, un thème qui hante toute l'œuvre d'Eastwood et lui donne une teinte quasi existentialiste. Elle repose aussi sur l'amour et l'amitié, les seuls liens valorisés par le réalisateur : dans Breezy (1973), Sur la route de Madison, Million Dollar Baby ou Gran Torino, ce sont eux qui permettent de triompher de l'aliénation familiale en évitant l'écueil de la solitude.

Lieu d'une émancipation individuelle, ces communautés libertariennes fournissent aussi un horizon d'utopie à une Amérique désorientée. On peut évoquer ici deux films que trois décennies séparent. Bronco Billy tout d'abord : il s'agit d'une farce à la façon de Frank Capra qui célèbre les idéalistes. À sa sortie, il a été interprété comme un éloge des valeurs conservatrices, mais le propos y est beaucoup plus ambitieux : le cœur du film, c'est la communauté formée autour d'un ancien vendeur de chaussures et repris de justice qui, à la fin du XX ${ }^{\mathrm{e}}$ siècle, va jusqu'au bout de son désir anachronique d'être un authentique cow-boy du Far West. S'il frôle souvent le ridicule, il réussit cependant à donner naissance à une famille recomposée qui comprend un ancien braqueur manchot, un vieil afro-américain qui pratiquait illégalement la médecine, un couple de faux Indiens qu'il a sauvé de l'alcoolisme, un jeune déserteur devenu un as du lasso et enfin une riche héritière désœuvrée. Cette troupe de sympathiques losers 
survit difficilement grâce à un Wild West Show et elle affronte les pires difficultés, la moindre n'étant pas l'incendie de son chapiteau, opportunément remplacé par un chapiteau tout neuf fait de cent drapeaux américains cousus les uns aux autres dans un hôpital psychiatrique par des aliénés. Derrière une apparence de fantaisie et de légèreté, c'est un appel libertaire à la relance du credo américain que délivre le cinéaste : l'Amérique peut se sauver en renouant avec l'esprit des pionniers et les valeurs des Founding Fathers. Petite Source, la fausse Indienne, exprime très bien cette adhésion à l'American Dream : «vous pouvez devenir qui vous voulez : il suffit de le vouloir », explique-t-elle. Trente ans plus tard, dans Gran Torino, Eastwood développe la même idée dans un contexte différent. Face aux mutations que connaît la société américaine, il soutient que le rêve américain est encore possible : si on lui apprend la liberté, la responsabilité individuelle, le sens de l'effort et la solidarité, un jeune Hmong peut devenir le digne héritier de l'américain old school qu'est Walt Kowalski. Comme l'affirme toujours le réalisateur, le rêve américain, entendu comme la possibilité « de mener à bien un projet dont vous avez rêvé », est « encore possible ici ${ }^{45}$ ». Tel est le message d'espoir qu'il livre dans ce film qui prend la forme d'un testament.

$$
* * *
$$

À l'encontre de la lecture reprise depuis quarante ans, nous suggérons de regarder le cinéma de Clint Eastwood non pas comme l'expression cinématographique du néoconservatisme, mais comme le produit d'une sensibilité libertarienne incontestable ${ }^{46}$. On se tromperait cependant à faire de ce réalisateur un disciple servile d'Ayn
Rand s'attachant à défendre la « vertu d'égoïsme ${ }^{47} »$. Comme le rappelle Ruwen Ogien, l'individualisme est en effet " parfaitement compatible avec des préférences altruistes et des engagements pour le bien commun ${ }^{48} »$. L'individualisme d'Eastwood ne doit pas être confondu avec la promotion d'un coupable solipsisme. Comme l'a bien relevé Jean Szlamowicz, le cinéaste prend clairement ses distances avec l'individualisme outré des trente dernières années pour illustrer la fécondité d'un «individualisme altruiste » prenant la forme d'un « hymne à la solidarité individuelle ${ }^{49} »$. Cette promotion de l'individualisme altruiste, déjà revendiqué par Albert Camus il y a plus d'un demi-siècle ${ }^{50}$, fait du cinéma d'Eastwood l'œuvre d'un humaniste et d'un moraliste raisonnant à l'aune de l'individu et de sa difficile liberté, mais sans négliger la question du rapport du je au nous, sans scinder le souci des autres et le souci de soi. Pour prendre la mesure de l'épaisseur du cinéma de Clint Eastwood, rien ne serait donc plus mutilant que de croire son auteur sur parole lorsqu'il affirme, en répétant sans le savoir le geste wébérien, qu'il faut dissocier le cinéaste et le politique. 
$\mathrm{N} \cdot \mathrm{O} \cdot \mathrm{T} \cdot \mathrm{E} \cdot \mathrm{S}$

1. "Clint Eastwood droit dans ses bottes », entretien donné à Valeurs actuelles, 24 février 2009.

2. Des exceptions existent néanmoins : cf. par exemple Stéphane Bouquet, Clint Fucking Eastwood, Paris, Caprici, 2012.

3. Théoricien marxiste italien, Antonio Gramsci (1891-1937) a forgé dans ses Carnets de prison le concept d' « hégémonie culturelle » afin d'expliquer le maintien, non-conforme aux prédictions de Marx, des États bourgeois. Pour les renverser, Gramsci soutenait qu'il fallait au préalable conquérir l'hégémonie culturelle par une stratégie métapolitique permettant de provoquer une transformation de la conscience des masses ouvrières. Cette idée a rapidement fleuri à gauche du spectre politique, mais elle a aussi été reprise à droite afin de lutter contre ce qui a été ensuite perçu comme une hégémonie culturelle de la gauche.

4. Cet article repose sur l'étude de l'ensemble de la filmographie de Clint Eastwood, à laquelle nous avons ajouté cinq longs-métrages dans la réalisation desquels il a joué un rôle décisif : Les proies (1971) - réal. D. Siegel ; L'inspecteur Harry (1971) - réal. D. Siegel ; Un frisson dans la nuit (1971); L'homme des hautes plaines (1973) ; Breezy (1973); Magnum Force (1973) - réal. T. Post ; La sanction (1975) ; Josey Wales horsla-loi (1976); L'épreuve de force (1977); Bronco Billy (1980); Firefox (1982); Honkytonk Man (1982); Le retour de l'inspecteur Harry (1983); La corde raide (1984) - réal. R. Tuggle; Pale Rider (1985); Le maître de guerre (1986) ; Bird (1988); Chasseur blanc, cœur noir (1990); La relève (1990); Impitoyable (1992); Un monde parfait (1993) ; Dans la ligne de mire (1994) réal. W. Petersen; Sur la route de Madison (1995); Les pleins pouvoirs (1997); Minuit dans le jardin du bien et du mal (1997) ; Jugé coupable (1999) ; Space cowboys (2000) ; Créance de sang (2002); Mystic River
(2003); Million Dollar Baby (2004); Mémoires de nos pères (2006) ; Lettres d'Iwo Jima (2006) ; L'échange (2008); Gran Torino (2008); Invictus (2009); Au-delà (2010); J. Edgar (2011); Une nouvelle chance (2012); Jersey Boys (2014).

5. On trouvera un panorama général dans Steven J. Ross, Hollywood Left and Right. How Movie Stars Shaped American Politics, Oxford, Oxford University Press, 2011.

6. La « trilogie du dollar» de Sergio Leone comprend : Pour une poignée de dollars (1964), Et pour quelques dollars de plus (1965), La Bon, la Brute et le Truand (1966).

7. L'idée d'une orientation « fascisante »-ou du moins autoritaire - de Clint Eastwood tire son origine de la célèbre critique de Pauline Kael, publiée dans The New Yorker le 15 janvier 1972 sous le titre « Saint Cop ». Pour une interprétation radicalement différente, on peut se reporter à Noël Simsolo, Clint Eastwood. Un passeur à Hollywood, Paris, Cahiers du Cinéma, 2003 et à Daniel Bénoliel, Clint Eastwood, Paris, Cahiers du Cinéma, 2012. De toute évidence, Magnum force, Le retour de l'inspecteur Harry et surtout Sur la corde raide se sont par la suite employés à lutter contre cette reductio ad fascistum qui a même parfois pris la forme d'une reductio ad hitlerum, la revue Positif qualifiant en janvier 1974 le deuxième film réalisé par Eastwood - L'homme des hautes plaines - de « Mein Kampf de l'Ouest ».

8. Ronald Reagan, discours d'investiture, 20 janvier 1981.

9. Patrick McGilligan, Clint Eastwood. Une légende, Paris, Nouveau Monde Éditions, 2008, p. 502. Pour une vision globale du cinéma américain durant cette décennie, on peut se reporter à Yannick Dahan, « Le cinéma américain sous Reagan », Cahiers d'histoire immédiate, n¹0, automne 1996, pp. 25-52.

10. Patrick McGilligan, Clint Eastwood, op. cit., p. 782. 
11. On parle de «westerns révisionnistes » pour qualifier les westerns modernes qui reposent sur une subversion des règles du genre posées par John Ford. Après les «westerns spaghetti » de Sergio Leone, les personnages centraux, plus ambigus, évoluent par-delà le bien et le mal ; les Indiens et les AfroAméricains sont décrits comme les victimes d'une construction nationale moins glorieuse ; la violence est omniprésente et les massacres sont légion. Concernant les westerns de Clint Eastwood, on parle aussi parfois de « westerns crépusculaires ».

12. Ajoutons qu'en octobre 2012, après son discours dit « de la chaise vide », Clint Eastwood a réitéré ce soutien en apparaissant dans une publicité télévisée financée par le Super-Pac American Crossroads et diffusée dans sept swing states.

13. Ce qui n'empêche pas la survie de cette critique erronée. Pour une occurrence récente, cf. Philippe Person, « Clint Eastwood a-t-il vraiment changé ? », Le Monde Diplomatique, juin 2009.

14. Akiva Gottlieb, « Last Man Standing : On Clint Eastwood », The Nation, 1 juin 2009 (consultable en ligne à l'adresse suivante : http://www.thenation.com/ article/last-man-standing-clint-eastwood\#).

15. Elle est cependant mieux connue depuis la parution des deux ouvrages fondamentaux de Sébastien Caré : La pensée libertarienne. Genèse, fondements et horizons d'une utopie libérale, Paris, PUF, 2009 et Les libertariens aux États-Unis. Sociologie d'un mouvement asocial, Rennes, PUR, 2011.

16. Dans le vocabulaire de Isaiah Berlin, ils défendent strictement la liberté négative. Cf. « Deux conceptions de la liberté » (1969), dans Éloge de la liberté, Paris, Calmann-Lévy, 1994.

17. C'est-à-dire, tel que le terme est entendu aux ÉtatsUnis, avec les progressistes.

18. Cf. par exemple l'entretien donné à Arthur Knight pour Playboy, en février 1974.
19. Entretien avec Jeff Dawson, « Dirty Harry comes clean », The Guardian, 6 juin 2008.

20. Entretien avec Mark Harris, GQ, septembre 2011 (consultable à l'adresse suivante : http://www.gq.com/ entertainment/movies-and-tv/201110/leonardodicaprio-clint-eastwood-gq-september-2011-coverstory-article).

21. Ce qui n'étonnera guère ceux qui ont vu Jugé coupable d'un côté ; Bird, Chasseur blanc, cœur noir et Invictus de l'autre.

22. Dans ses films, les prêtres sont d'ailleurs souvent des personnages perfides et retors, comme le prêtre de Jugé coupable qui est prêt à tout - même à décrire avec sadisme l'horreur du supplice à venir - pour soutirer une confession à un condamné à mort innocent. On ne trouvera un personnage " positif » de prêtre que tardivement dans son œuvre, dans Gran Torino : il faut dire qu'il a appris la « vraie vie » au contact de Walt Kowalski.

23. Il s'agit du slogan officiel du Libertarian Party, qui s'est autoproclamé «le parti du principe ».

24. Pour Seymour Martin Lipset, ce credo combine cinq principes : la liberté, l'égalité des chances, l'individualisme, le populisme et le laisser-faire. Cf. American Exceptionalism : A Double-Edged Sword, New York, W. W. Norton, 1997.

25. Propos cité par Michael Henry Wilson, Eastwood par Eastwood, Paris, Cahiers du Cinéma, 2010, p. 71. 26. Un exemple récent : le livre de Samuel P. Huntington, Qui sommes-nous? Identité nationale et choc des cultures, Paris, Odile Jacob, 2004.

27. Sur ce concept, cf. George L. Mosse, La brutalisation des sociétés européennes. De la Grande Guerre au totalitarisme, Paris, Hachette, 2000.

28. Une idée présente aussi bien chez Sigmund Freud (Totem et tabou, Paris, Payot, 2004) que chez René Girard (La violence et le sacré, Paris, Fayard, 1972). 29. Ce traitement de la violence fait penser à celui 
choisi vingt ans plus tard par David Cronenberg dans A History of Violence (2005).

30. Clint Eastwood fait aussi parfois référence à l'assassinat du président Lincoln - comme Dans la ligne de mire -, ce qui est sans doute une manière d'établir une continuité entre l'Amérique du XIX ${ }^{\mathrm{e}}$ siècle et celle du $X X X^{\mathrm{e}}$ siècle.

31. Lord Acton, Historical Essays and Studies, London, MacMillan \& Co, 1907.

32. Entretien donné à Parade Magazine, 12 janvier 1997.

33. Propos cité par Michael Henry Wilson, Eastwood par Eastwood, op. cit., p. 50.

34. Cf. par exemple Howard Roark dans Ayn Rand, $L a$ source vive, Paris, Plon, 1999 (1943).

35. Sur cette distinction, cf. Lucien Jaume, L'individu effacé ou le paradoxe du libéralisme français, Paris, Fayard, 1998.

36. Propos cité par Michael Henry Wilson, Eastwood par Eastwood, op. cit., p. 78.

37. Montesquieu, De l'esprit des lois, Paris, Flammarion, 1993 (1748).

38. Sur les fonctions politiques positives de l'utopie, cf. Paul Ricœur, L'idéologie et l'utopie, Paris, Seuil, 2005.

39. Ferdinand Tönnies, Communauté et société. Catégories fondamentales de la sociologie pure, Paris, PUF, 2010 (1887).

40. Émile Durkheim, « Communauté et société selon Tönnies » (1889), dans Textes 1. Éléments d'une théorie sociale, Paris, Éditions de Minuit, 1975, p. 383. 41. On emprunte cette expression à John Stuart Mill. Cf. De la liberté, Paris, Gallimard, 1990 (1859).

42. Propos cités par Michael Henry Wilson, Eastwood par Eastwood, op. cit., p. 71.

43. On pense ici à Atlas Shrugged, publié par Rand en 1957, dont le récit se déploie autour de la communauté « sécessionniste » fondée par John Galt.
44. Propos cité par Michael Henry Wilson, Eastwood par Eastwood, op. cit., p. 62.

45. Ibid, p. 198.

46. Les néoconservateurs ont compris tardivement qu'il n'était pas des leurs : alors qu'ils vénéraient Harry le charognard dans les années 1980, ils ont fini par renier Eastwood dix ans plus tard, après Un monde parfait, au motif qu'il serait devenu un partisan de la conception sociale de l'origine de la criminalité, donc une sorte de dangereux « gauchiste ». On peut comparer deux articles de Richard Grenier pour prendre conscience de l'évolution de la position des neocons : «The World's Favorite Movie Star », Commentary, mars 1984 et «Clint Eastwood Goes PC », Commentary, mars 1994. 47. Ayn Rand, La vertu d'égoïsme, Paris, Les Belles Lettres, 2008.

48. Ruwen Ogien, L'État nous rend-il meilleurs? Essai sur la liberté politique, Paris, Gallimard, 2013, p. 126. 49. Jean Szlamowicz, « Le discours cinématographique de Clint Eastwood : individualisme et humanisme », Raisons politiques, 2010/2, n³8, p. 58.

50. Albert Camus, L'homme révolté, Paris, Gallimard, 1985 (1951). 


\section{$R \cdot E \cdot S \cdot U \cdot M \cdot E ́$}

Cet article défend une lecture hétérodoxe du cinéma de Clint Eastwood, conçu comme étant un vecteur aussi discret qu'efficace d'une philosophie politique radicale : le libertarianisme. Cette thèse s'oppose aux deux interprétations dominantes de l'œuvre du réalisateur. D'un côté, la critique mainstream qui trouve dans ses films une promotion et une légitimation habiles des valeurs du néoconservatisme américain. De l'autre, la lecture esthète qui, prolongeant le discours du réalisateur lui-même, met en avant une pure œuvre artistique dénuée de portée politique. Après un retour sur l'itinéraire politique atypique d'Eastwood, on montre que son cinéma est hanté par une interrogation sur la violence et sur le destin de l'Amérique fortement teintée par l'anarchisme individualiste. Son œuvre doit alors être analysée comme une contribution libertaire à la relance du credo américain dans un contexte de désappointement idéologique, auquel elle entend répondre en proposant un horizon mêlant utopies communautaires et individualisme altruiste.

\footnotetext{
Abstract

This article defends an heterodox interpretation of Clint Eastwood's cinema, perceived as a discreet and efficient vector of a radical political philosophy: libertarianism. This analysis is against the two main interpretations of the director's work. On the one hand, the mainstream criticism which finds in its movies a clever promotion and legitimation of American neoconservatism values. On the other hand, the aesthete interpretation which points out a pure artistic work without any political significance, in line with the director's
}

words. After a come back on Eastwood's unusual political itinerary, we show that its cinema is haunted by a question about violence and about America's destiny heavily influenced by individualist anarchism. Thus, its work has to be interpreted as a libertarian contribution to the revival of American creed in a context of ideological disappointment, to which it intends to respond by offering an horizon blending community utopia and altruistic individualism. 